\title{
Deployment-based Solution for Prolonging Network Lifetime in Sensor Networks
}

\author{
Sonia Hashish and Ahmed Karmouch \\ School of Information Technology and Engineering(SITE) \\ University of Ottawa-Canada \\ \{shashish,karmouch\}@site.uottawa.ca
}

\begin{abstract}
Enhancing sensor network lifetime is an important research topic for wireless sensor networks. In this paper, we introduce a solution for prolonging the lifetime of sensor networks that is based on a deployment strategy. In our proposal, data traffic is directed away from the network center toward the network peripheral where sinks would be initially deployed. Sinks stay stationary while collecting the data reports that travel over the network perimeter toward them. Eventually perimeter nodes would be exposed to a peeling phenomenon which results in partitioning one or more sinks from their one-hop neighbors. The partitioned sinks move discrete steps following the direction of the progressive peeling The mechanism maintains the network connectivity and delays the occurrence of partition. The performance of the proposed protocol is evaluated using intensive simulations. The results show the efficiency (in terms of both reliability and connectivity) of our deployment strategy with the associated data collection protocol. Keywords: Sensor networks, Data collection, Mobile sinks, Deployment.
\end{abstract}

\section{Introduction}

Maximizing the lifetime of sensor network would convert the theoretical achievements in sensor network research to successful real world deployed networks.

One of the reasons that threaten the lifetime of sensor networks is the energy depletion of nodes located around the data collector nodes (usually called sinks). Such energy depletion forms what is called an energy hole around the data collector [1]. Energy hole is most likely unavoidable in networks depend on stationary-based sinks. This is definitely true for networks with uniform node distribution and uniform data reporting. Since data travels to the collector using multi-hop communication model, nodes located near the collectors are exposed to heavier load. Such nodes lose their energy resources faster than others and an early sink-network partition occurs.

Maximizing sensor network lifetime is considered as an optimization problem in linear programming. It has been proved to be NP-hard [2]. A pproximation algorithms are developed to provide efficient solutions under certain settings. The dynamicity and the frequent updates in the network topology are usually unpredictable which reduces the efficiency of such solutions in many cases. Many other practical solutions have been considered within the literature to minimize the tendency of the nodes located near sinks to form an early partition. Examples of these solutions are (1) replacing the stationary sinks with mobile ones, (2) considering the dynamic clustering of the network, (3) intentionally performing non-uniform (controlled) node 
distribution over the coverage area. Although effective solutions, they have some limitations and constraints in real world deployments.

Using different clustering techniques to enhance the network performance has been known for long time in the area of ad-hoc networks as well as sensor networks. However, clustering algorithms are expensive in terms of their communication cost. They require extensive overhead that could contribute to drain the resources of sensor networks [3, 4].

Exploiting sink mobility to enhance sensor network lifetime raises many new problems related to the mobility. Examples are: what is the best mobility pattern that would be implied and how to lessen the interference of the mobility trajectories (in case of multiple mobile sinks) [5]. In addition to the latency problem that results from the difference between the speed of moving the sink to collect the data versus the multi-hop transmissions of the data. Some techniques imply infrequent mobility [6]. Mobile sinks infrequently move to balance the load among nodes and a multi-hop wireless transmission is used as the main regime of data transportation.

On the other hand, intentionally performing non-uniform node distribution [7] is problematic and difficult to be achieved in real world deployments. This is due to the expected large number of sensor nodes that usually should be randomly deployed within the coverage area [8].

In this paper we introduce a solution that maximizes the sensor network lifetime. The proposed solution combines the benefits of using multiple-mobile sinks and the non-uniform node distribution (without performing non-uniform node distribution). Our proposed protocol reduces the problems associated with the above mechanisms. In our proposal, data traffic is directed away from the network center toward the network peripheral where sinks would be initially deployed. Sinks stay stationary while collecting the data reports that travel over the perimeter toward them. Eventually perimeter nodes at the network peripheral would be exposed to a peeling phenomenon (have their energy depleted exposing other inner nodes to be perimeter) which results in partitioning one or more sinks from their one-hop neighbors. The partitioned sinks move discrete steps following the direction of the progressive peeling and the connectivity is re-established. The protocol is totally dynamic. The overhead of exchanging topology updates messages associated with the mobility is not required. We show that our solution leads to a sub-optimal energy balancing through the network. The balance of both load and energy consumption leads to an expansion of the network lifetime. The performance of the protocol is shown by intensive simulations that consider realistic conditions of the underlying network settings. The results show the efficiency of the protocol in expanding the network lifetime, minimizing overhead associated with sink-mobility, and achieving high degree of reliability.

The rest of this paper is organized as following Section 2 discusses current related work. Section 3 explains our assumptions about the underlying network model. Section 4 describes the design details of the proposed boundary-peeling data collection protocol. Section 5 summarizes the performance of the approach. Discussions are given in section 6. Section 7 provides a brief conclusion.

\section{Related work}

Extensive work has been proposed into the literature to enhance the sensor network lifetime using different conceptual approaches. Due to the limited space we only list 
those we believe they are the most related to our work. Routing mechanisms that aim at balancing energy consumption and load through the network have been taken a considerable attention. Some of these protocols consider mobile sinks. Their main concern is how to route toward the mobile sink rather than how to exploit the mobility of sinks to enhance the lifetime of the network. A good survey of such mechanisms is [9]. The guidelines study of energy balancing that lead to enhancing the lifetime in sensor networks which consider static sinks is given in [10]. Authors conclude that the optimal energy balancing is impossible under some conditions. They also suggest a solution based on training the network into coronas with equal widths. The widths of such coronas should be determined based on the energy loss factor.

Many approximation algorithms have been developed for maximizing the lifetime of the network. Most of these algorithms are interested in the behavior of sensor node rather the than sink node. One of the proposals that consider the behavior of the sink node is found in [11]. Authors determine the location of the base station node (the data collector) that could result in maximizing the lifetime. The algorithm considers the routing strategy that would be used but no mobility is considered.

Exploiting mobility to extend network lifetime has been introduced recently into wireless sensor networks. More and more work has been devoted to address the effect of sink mobility on enhancing the performance of the network. In some proposals [1214] mobile sinks would be used as transportation units where they move to collect the data from the stationary nodes. Such approaches result in significant energy saving but the latency problem is the main concern. Other approaches consider mobility of the base station as an assisted facility to improve the network performance $[5,6,15]$. In such assisted mobility protocols, the main regime of data transfer is the wireless multi-hop transmissions; mobility helps reducing hot spots and increases the load balancing through the nodes. So these approaches are always associated with data collection mechanisms that maximize the benefits of the mobile sinks.

Our work belongs to the assisted mobility protocols. The most relevant work to our work is that in [15]. The authors analytically prove that the optimal mobility trajectory in case of circular networks is where the mobile sink moves around the network peripheral. Our work in this paper complements such analytical foundations and inspired by our previous work in the area of data dissemination [16]. Authors in [15] suggest that the mobile sink constructs a global routing tree based on the shortest path while moving. The peripheral nodes forward their data using trajectory forwarding routing follows the mobility trajectory (in that case a circle). The scheme tends to be less adaptable to the topology irregularities. Both shortest path tree and trajectory forwarding are proactive mechanisms which require extra communication overhead to keep their performance high.

Joint mobility strategy is also suggested in [6] where a routing scheme is proposed to route the data to a single mobile sink. The sink moves around the network peripheral. Authors propose that each data collection round is preceded by a sampling round. In a sampling round, the sink stops at certain anchor positions and determines the optimal visiting time by sampling the global power consumption. The visiting time is then applied to control the movement of the sink while in the data collection round. Both [15] and [6] consider a single mobile sink. Multiple-mobile sinks raise the fact that coordination is required to keep the "positive effect" of the mobility trajectories interference on the network performance. Authors in [5] provide multiple algorithms to solve such problem.

Our work differentiates itself by considering multi-mobile sinks with mobility pattern that inherently simple and non-interfereable. The protocol efficiently adapts to the topological changes. It doesn't require any global knowledge that should be 
collected in a centralized manner. It also works for any network topology whether regular or irregular.

\section{Network Model}

Network infrastructure: We assume a two-dimensional terrain area. The distribution of the nodes within the terrain area is uniformly random. Sensor nodes have the same capabilities in communication, computation and storage. Each sensor node knows the coordinates of its location using either GPS or any existing localized techniques [17]. Two nodes are considered to be neighbors if each of them is located in the transmission range of the other. Sinks or data collectors are special nodes with no restriction in their energy resources or their communications capabilities. We assume multiple of such special nodes are initially placed arbitrary at the network peripheral. Data collector nodes could be the end-points of the data collection process or they could further forward the collected data to other remote site over long wireless radio.

Energy and traffic models: We assume that all sensors have the same initial energy rate $e$. Although sensors use their energy while they are transmitting, receiving and sensing, we assume that the transmission process is the dominant factor of the energy depletion [17]. We use the uniform data reporting model where each sensor $i$ generates data with fixed rate $\mu_{\mathrm{i}}$. Each node consumes a unit of energy to transmit one unit of data. So the number of transmissions performed by each node is a good indicator of its energy consumption rate. No data aggregation is considered. Each node transmits its own data and any data units that it receives to forward on behalf of other nodes. Sensor nodes complete their task when they forward their data to any of the data collector nodes.

Mobility Model: we assume that each sink has a controlled mobility facility [18]. Sinks travel using a discrete mobility pattern $M$ where the movement is performed in steps and only under some conditions (as we will explain in next section).

\subsection{Balancing the energy expenditure}

In this section we show that our protocol that we describe in next subsections leads to a sub-optimal energy balancing through the network as defined in [7]. Authors in [7] consider a circular network model where the network area $A$ is partitioned into coronas with equal widths (fig. 1). They prove that, if the sink is located at the center of the network $c$ and the nodes are distributed non-uniformly according to the following rule: $N_{i} / N_{i+1}=N_{i+1} / N_{i+2}=\ldots=q$ (where $q>1$ and $N_{i}$ is the number of nodes in area $A_{i}$ known as corona $i$ ), a suboptimal energy balancing could be achieved. In other words if the number of nodes in corona $i$ which is nearer to the sink is larger than the adjacent corona $i+1$ by a factor $q$ a suboptimal energy balancing is achieved. The authors define the suboptimal energy balancing as the ability to balance the energy among all the inner parts of the network except the outmost one.

Distributing a large number of nodes within the coverage area using the above rule could be difficult in real world deployment. We prove that without the need for such controlled distribution, we could achieve a similar sub-optimality. This could be achieved by considering deploying the data collector nodes at the network peripheral. In addition to such exterior deployment, a data dissemination strategy that allows the 
direction of traffic to be reversed (from inner parts with smaller number of nodes to the outer parts with larger number of nodes) should be implied.

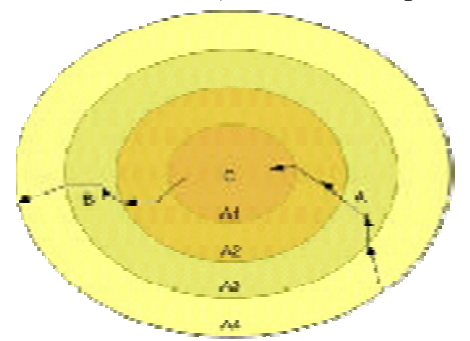

Fig. 1. Area -Dissemination direction relationship. The network is partitioned into number of coronas. Paths $A, B$ represent two directions of traffic. $C$ represents the network center.

In fig. 1 path $A$ represents one possible data traffic paths from outer corona to inner one where the sink is assumed to be located at the network center. Non-uniform node distribution is represented by darken the corona (i.e. the darker the corona the increased number of nodes) so the number of nodes should be increased with geometric proportion from the outer parts to the inner ones.

In our deployment model no controlled node distribution is performed. Instead; the direction of the traffic is reversed. Considering the same network model in fig 1, path $B$ represents a possible traffic path. Assuming uniform node density $D=N / A$ where

$$
\begin{gathered}
D_{1}=D_{2}=,,,=D_{n}=N_{i} / A_{i} \quad, 1 \leq \mathrm{i} \leq \mathrm{n} \\
\text { Intuitively } A_{1}<A_{2}<\ldots<A_{i} \text { so } N_{1}<N_{2}<\ldots<N_{i} \\
N_{i+1} / N_{i}=N_{i} / N_{i-1}=\ldots=q \text { and } q>1
\end{gathered}
$$

This means that with uniform node distribution, corona $i+1$ has number of nodes larger than the adjacent corona $i$ (which is nearer to the network center). This is intuitively enforced by the underlying area to be covered by node distribution. So if the traffic is reversed toward the outer corona where data collector nodes would be deployed we could get the same benefit without intentionally perform a controlled non-uniform node distribution.

\subsection{Data dissemination strategy}

Reversing the direction of the data traffic from inner parts to outer parts and deploying the data collector nodes at the network peripheral are not enough to get the benefit of the sub-optimal balancing of the energy. Without a data dissemination strategy that could take advantage of the previous consideration, the external sink deployment imposes higher communication costs than the centric-sink model. Implicitly communication paths would be longer in terms of hops count. Nodes around the data collector have to carry the data from relatively larger number of far nodes. These nodes are exposed to drain their energy resources and die faster than others forming an early partition. So a data dissemination strategy is required to take advantages of the sub-optimal energy balance that could be obtained by reversing the direction of the traffic. In the following subsection we describe our boundary-peeling data collection protocol that works for both regular (circular, grid) and irregular network topologies. So the network model in fig 1 is relaxed to the general network 
model described in section 3. The protocol aims to prolong the network lifetime defined as the time until a network partition occurs [2]. Partition could be either a sink-network partition or internal partition where group of nodes form an isolated island.

\section{Boundary-Peeling Data Collection Protocol}

The proposed data collection protocol consists of three phases: initial, continuous and conditional one. Fig 2, shows the conceptual steps of the data collection protocol.

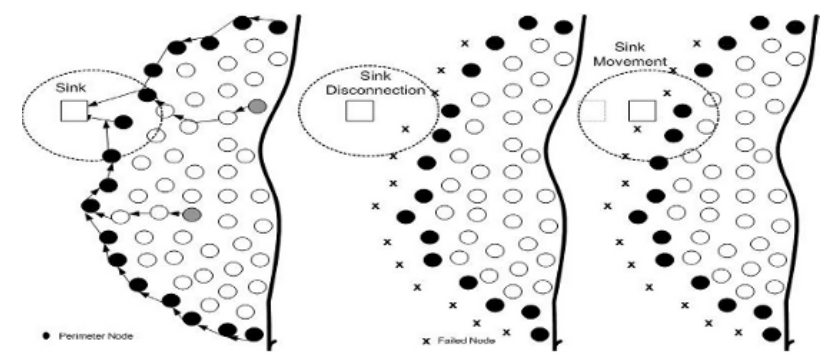

Fig. 2. The boundary-peeling data collection process. (a) The network boundary is recognized and data is directed to the boundary where it travels to the sink. (b) Sink-network partition. (c) Sink movement toward the network center.

\subsection{Phase I: Topology recognition}

This phase is performed once at the setup time. It consists of three steps. The boundary of the network is recognized at the first step. This step allows each boundary node to know that it is indeed a boundary node. The second step is the computation of the virtual centroid (v-center) of the network. This is done offline at one sink (if multiple exist) elected to perform the computation. At the third step the vcenter is broadcasted to all nodes. Each node uses the v-center to learn its allowable forwarding directions (to be used in phase II). If the center of the network is known in advance as in the case of the circular network model, it could be used directly (so we use the terms $v$-center and network center interchangeably). In reality, networks are neither circular nor regular. Networks with irregular topologies are important class of sensor networks.

We use the BoundHole algorithm [19] to define the boundary of the network and allow perimeter nodes to know that they are located at the network boundary. The algorithm was developed to detect the holes within the sensor field. It inherently identifies the outer boundary of the network. Peripheral sinks cooperatively implement the Boundhole at the initial phase. Assuming a total of $m$ sinks $S_{0}, S_{1}, \ldots$, $S_{m-l}$, we divide the boundary into $m$ boundary segments associated with the $m$ sinks. Each segment starts at one sink and ends at the next sink in a counterclockwise direction. So the outer boundary is represented by the following sequence: $S_{0}, p_{1}, p_{2} \ldots$, $p_{i}, S_{1,} p_{i+1}, p_{i+2 \ldots} \ldots p_{k}, \mathrm{~S}_{2,} p_{k+1} \ldots S_{m-1 \ldots} p_{j}, S_{0}$. where $p$ 's are the sink-to-sink nodes located at the outer boundary. Each perimeter node $p$ keeps information about its upstream and downstream p's nodes in addition to counters (registering its hop-count) to each end-sink. A node also creates a pointer to its parent (upstream) node toward 
the closest end sink. Perimeter nodes would exhibit the heavier load amongst the network nodes. Frequently such nodes would lose their energy and die exposing other interior nodes to be perimeter. The algorithm is supported by a local maintenance process (at level of one-hop neighbors) that allows the replacement of the dead perimeter nodes by fresh ones. Such local maintenance process allows dynamic coping with topological changes affecting the outer boundary and capturing the continuous updates.

\section{Computation of virtual centriod}

To compute the $v$-center $c$, we consider the network as a closed polygon in which a finite set of perimeter nodes makes up a set of virtual vertices, or v-points. The $v$ center is defined as the median of this set. The concept of the $v$-center provides only an approximate solution as the network is not really a polygon with known vertices. The $v$-points can be selected using a threshold distance value ( $h$ number of hops) as following: once the network perimeter is marked, perimeter nodes that satisfy $k h$ distance (where $\mathrm{k}=1,2,3, .$. , etc.) to the sink that is elected to perform the computation, report their coordinates. The sink collects the positions of the v-points and determines the coordinates $\left(x_{c}, \mathrm{y}_{c}\right)$ of $c$ as in (4) where $A$ is the virtual network area using the selected v-points [20]. The v-points could also be selected as the ends and middle of each boundary segment. We note that the smaller the value of $h$, the more precise the $v$-center.

$$
x_{c}=\frac{1}{6 A} \sum_{i=0}^{K-1}\left(x_{i}+x_{i+1}\right)\left(x_{i} y_{i+1}-x_{i+1} y_{i}\right), y_{c=} \frac{1}{6 A} \sum_{i=0}^{K-1}\left(y_{i}+y_{i+1}\right)\left(x_{i} y_{i+1}-x_{i+1} y_{i}\right)
$$

\section{Forwarding directions}

The $v$-center $c$ computed at the previous step acts as a center of a disperse force that directs the data away from it toward the network peripheral. Each node learns its allowable forwarding directions (out of four basic directions Up, Down, Left, Right) according to its location with respect to $c$. For each node, the four basic directions are represented by four abstract reference points on its communication circumference (the circle with the node as the center and the communication radius as the radius). The allowable directions are those that direct the data away-from the v-center. For example, if the location of the $v$-center $c$ with respect to node $n_{l}$ is at up-right corner of an imaginary rectangle, $n_{1}$ excludes both Up and Right directions. $n_{1}$ considers the remaining directions (Left and Down) as its allowable forwarding directions. The node alternatively selects its current forwarding direction (the direction where it should transmit its data) out of them.

\subsection{Phase II: Data Forwarding}

The data forwarding phase is a continuous phase Interior (non-perimeter) nodes forward their data toward the network perimeter. The node selects the current direction of data forwarding and transmits the data to the next forwarder in that direction. The next forwarder is the neighbor closest to the reference point of the chosen direction among those closer to that point than the node itself. The process continues until the data arrives at any perimeter node. Data arrived at the perimeter region travels the perimeter toward the closest sink. 


\subsection{Phase III: Sink Mobility}

The mobility phase is a conditional phase sinks stay stationary at their initial deployment locations until one of the movement conditions is satisfied. Sinks continuously validate the conditions and make the movement decision when required. A condition could be (a) a sink is exposed to a full partition at its current location, (b) the current number of its one-hop neighbors is less than a threshold value and (c) The rate of receiving data at the current location is less than an expected threshold value. The movement pattern is such that the sink moves discrete steps toward the network $v$-center. So the $v$-center acts as a center of a pulling force that attracts the moving sinks (in contrast to the "away-from-centroid" dissemination strategy that is assumed for internal data propagation). The moving sink checks the validity of the moving condition in each step and moves to regain its initial situation. In each step, the sink moves a progressive distance equal to its transmission range in the direction of the line joining the current location of the sink to the network center. Once the movement action is completed, the sink informs its current one-hop neighbors of its presence in their transmission region. It sends them an explore message and collects their replies. The sink reassigns the role of perimeter neighbors among them and sends a glueboundary message. The perimeter nodes locally glue the boundary within their segment and adjust their hop-count toward the sink. They also modify their pointers to the upstream neighbors toward the closest sink if required. This mechanism maintains the connectivity of sinks to the network boundary.

\section{Performance Evaluation}

We evaluated our protocol using the wireless sensor network simulator (WSNS) described in [21]. Two categories of experiments are performed to test the behavior of the protocol in both dense and sparse networks. We used network terrain dimensions of $400 \mathrm{~m} \times 400 \mathrm{~m}$ with sensor densities ranging from 200 to 400 nodes (randomly distributed). Different radio ranges $\mathrm{T}_{\mathrm{x}}$ (ranging from $60 \mathrm{~m}$ to $90 \mathrm{~m}$ ) are used to maintain the connectivity of the network with the changed densities. Experiments are conducted with different number of sinks deployed dynamically at the network peripheral. In all experiments, the initial energy of sensor nodes is set to 100 units. We assume that each data transmission would consume one unit of energy. For simplicity, the third mobility condition mentioned in the pervious section is not simulated and we stop the simulation when a partition occurs.

A third category of experiments is conducted with centric-sink network model and the performance is compared to our model. In centric-sink model, a single sink is located at the center of the network and the shortest path routing SPR is applied as the underlying routing protocol (this model is considered as an ideal model in $[6,7,10$, 15]). Since nodes are stationary and no underlying MAC protocol is considered, Routing tables of the SPR are determined at the initial phase. We then allow a control packets transmission session every simulation round (to update the routing information and cope with routing failures due to the increase in the number of dead nodes). We assume that each node consumes 0.5 unit of energy to transmit one control message. Results from the simulation are collected at different rounds. Each round is equivalent to 2 data transmission sessions and one control transmission session. To ensure consistency, all the plotted results are the average of 5 runs of each experiment. 


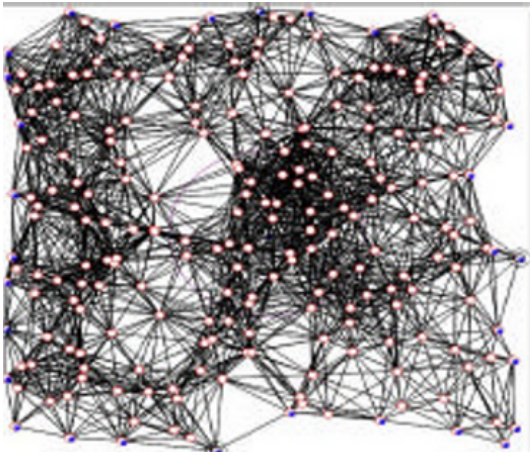

(a)

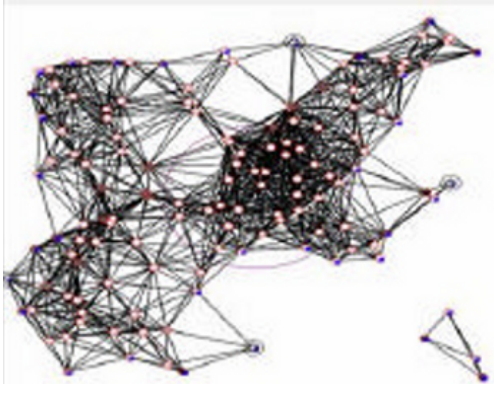

(b)

Fig. 3. Simulation snapshots at different simulation time, (a) the initial topology, 4 sinks are deployed at the network peripheral (nodes with double circles), perimeter nodes are recognized and each node is marked with small dark rectangular, (b) The network partition (some nodes form an isolated island the simulation stops at this point).

Fig 3, shows snapshots of simulation runs at different simulation rounds. It shows the updates of the network boundary (that we call the peeling phenomenon) in a dense network scenario. It also illustrates the movement of sinks toward the network center (sinks are not necessary moving simultaneously).

For each configuration scenario, the level of each node with respect to the network center is determined Level 1 nodes are those located at distance less than $T_{x}$ with respect to the network center $c$. Level 2 nodes are those located at distance larger than $\mathrm{T}_{\mathrm{x}}$ and smaller than $2 \mathrm{~T}_{\mathrm{x}} \mathrm{w}$ ith respect to $c$ and so on.

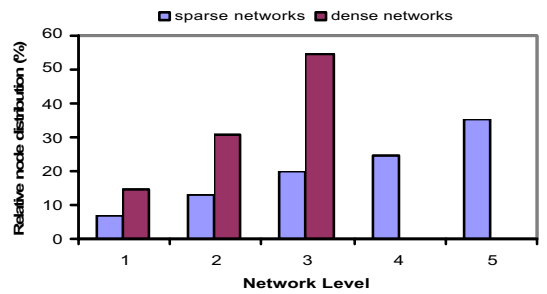

Fig. 4. Relative node distribution

Fig 4 shows relative node densities of different network layers for both dense and sparse network configurations. Layer one is the nearest one to the network center. In dense networks, the nodes are separated over three layers. In sparse networks, nodes are separated over 5 layers. The relative densities are shown in fig 4 .

To show how our deployment model with the associated data collection protocol results in load balancing through the network, the average load at each network level is measured and depicted in fig 5. The figure shows that our protocol definitely balances the average load at each network layer except the outmost one (network level 5 in sparse settings and 3 in dense settings). The figure also shows that increasing the number of peripheral sinks results in decreasing the average load at the outmost layer. The inner layers would approximately exhibit the same average load. In the case of centric-sink model with shortest path routing, the innermost layer (level 1) is exhibiting the higher load amongst others. The gradual increase in the average load from outmost layer to innermost layer is higher than that exhibited by our model for both sparse and dense networks. 

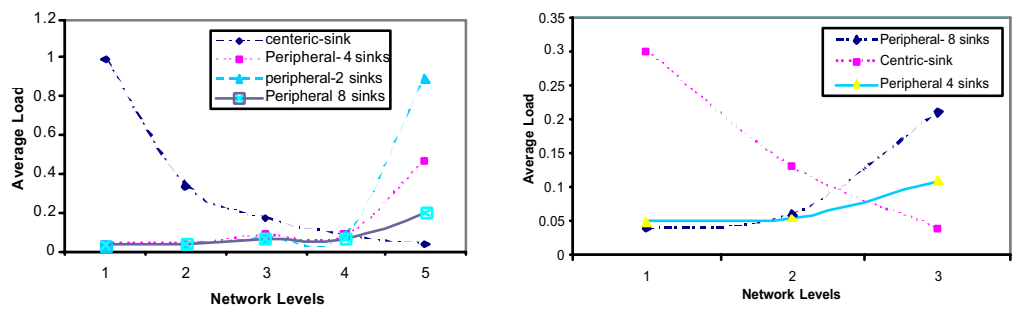

Fig. 5. Comparison of average load at each network level in case of sparse network (Left) and dense network (right).

The number of dead nodes is measured at each simulation round and the average rate of dead nodes is depicted in fig 6 .
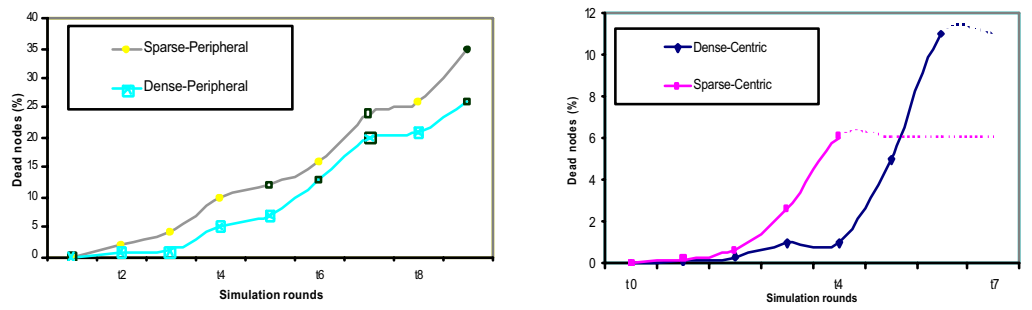

Fig. 6. The rate of dead nodes at different simulation rounds. (Left) Small squares explain the points of movement of one or more sink s and the slid black circle explains the point of partition. (Right) Dotted segments explain the network partition.

The figure shows that our protocol exhibits a higher rate of dead nodes than that exhibited in the case of centric-sink model. Most of these nodes are separated over the network boundary which reduces the negative effect of their early death. Fig. 6 also illustrates the simulation rounds at which the partition occurs. The results are the average results obtained for different network settings where 10 simulation rounds are allowed per each setting. In sparse settings, our model maintains the network connectivity twice the time exhibited using the centric-model. In dense settings, the partition is more delayed than in the case of sparse networks for both models. The partition occurs around the seventh simulation round in case of centric-model while no partition appeared using our model over the 10 simulation rounds.

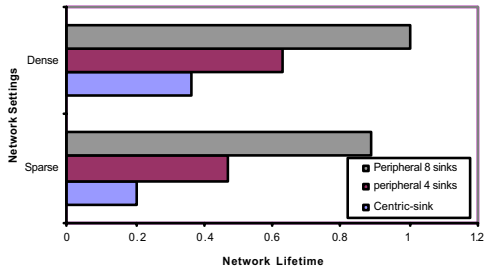

(a)

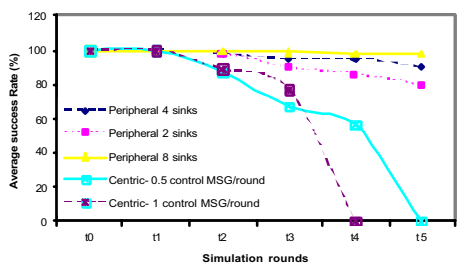

(b)

Fig. 7. Normalized network lifetime and the average success rate

The normalized network lifetime is shown in fig. (7-a). The figure shows the relative increase in the network lifetime achieved by our protocol. It also shows the 
effect of increasing the number of peripheral sinks. We also measure the success rate of both models in the two network categories. The success rate measures the capability of the live nodes to send their data successfully to the sink. Fig. (7-b) shows the average results obtained for both dense and sparse configurations. Both models demonstrate $100 \%$ success rate at the early simulation rounds. Our deployment model with the associated data collection protocol keeps the success rate high during the whole simulation (in both network configurations). The figure shows that at the time a partition occurs the decrease in the success rate is very small. Most of the live nodes are able to send their data successfully to the sink. Fig (3-b) shows an example where only a small group of the live nodes forms an isolated island.

The success rate in case of Centric-sink model heavily depends on the transmission rate of the control messages. Such messages provide freshness of the routing tables. Increasing the rate of control messages increases the success rate yet decreases the network lifetime. This is due to increasing the energy consumption rate at each node which in turn fastens the network partition. When the sink is partitioned from the network the success rate drops to zero even thought a large number of nodes could be alive.

\section{Discussions}

The proposed deployment model with the associated data collection protocol reduces the negative effect of the peeling phenomenon. The direction of the peeling is from outmost layers to inner ones (external peeling). Since the proposed mobility strategy allows the movement of sinks to follow the direction of the progressive peeling (toward the network center i.e. from outmost to innermost), sinks are allowed to be approximately always connected. Even at the time partition occurs as in fig (3-b) a large part of the network is still connected to one or more sinks and the network is still operable. This raises a question about the coverage percentage that could be tolerated by the application. This feature of our protocol increases the utilization of the network resources. In the case of centric-sink the peeling phenomenon also occurs yet the direction of such peeling is toward the outmost layers. Such internal peeling results in early partition of the sink. The outmost layers could be thought of as guard layers that protect the inner layers If the deployment is planned such that the outer layers are assumed to be additional layers to the core network layers, exposing such layers to a peeling would not affect the interested coverage area. The network lifetime would be increasing proportional to the number of nodes in the outer layers. The coverage would be maintained according to the application requirements.

\section{Conclusion and Future work}

In this paper, we described the boundary-peeling data collection protocol and the associated sink-deployment model. The contributions of our solution includes the following: (i) it combines the benefits of using multiple and mobile sinks without introducing the problems associated with the sink mobility, (ii) It adapts to the topological changes in an efficient and dynamic manner with no overhead to exchange topological updates messages, and (iii) it balances the load among the nodes dynamically. The protocol with the associated deployment model is highly reliable. It is able to maintain the network connectivity as long as the coverage percentage is 
tolerated by the application. Further investigations of guard regions and the adaptive mobility are left for future work. Our sights are set initially on the development of policy-based sink mobility that adapts to the underlying sensing field conditions.

\section{Reference s}

1. Li, J., Mohapatra, P.: An analytical model for the energy hole problem in many-to-one sensor networks In: Proc. of IEEE VT C, Fall 2005.

2. Joongseok Park, Sartaj Sahni: An Online Heuristic for Maximum Lifetime Routing In Wireless Sensor Networks IEEE Trans. Computers 55(8): 1048-1056 (2006).

3. Soro, S., Heinzelman, W.: Prolonging the lifetime of wireless sensor networks via un equal clustering In: Proc. of the19th IEEE International Parallel and Distributed Processing Symposium (IPDPS), 2005.

4. Li, C. F., Ye, M., Chen, G., Wu, J.: An energy-efficient unequal clustering mechanism for wireless sensor networks In: Proc. of the 2nd IEEE International Conference on Mobile Adhoc and Sensor Systems (MASS), Washington, DC, Nov. 2005.

5. Athanasios, I., Rolim, J.: Fast and Energy Efficient Sensor Data Collection by Multiple Mobile Sinks In: ACM MobiWac 2007, Crete, Greece, October 22, 2007.

6. Luo, J., Panchard, J., Piorkowski, M., Grossglauser, M., Hubaux, J. P.: Mobiroute: Routing towards a mobile sink for improving lifetime in sensor networks. In: IEEE DCOSS, volume LNCS 4026, pages 480--497, 2006.

7. Xiaobing Wu, Guihai Chen, and Sajal Das, : On the Energy Hole Problem of Non-uniform Node Distribution in Wireless Sensor Networks. In: Proceedings of IEEE MASS 2006, October 2006.

8. Akyildiz, I. f., et al.: Wireless sensor networks: a survey. Computer Networks, Vol. 38, March 2002, pp. 393- 422.

9. Al-Karaki, J. N., Kamal, A. E.: Routing techniques in wireless sensor networks: a survey. IEEE Wireless Communications, vol. 11, no. 6, pp. 6-28, Dec. 2004.

10. Olariu, S., Stojmenovic, I.: Design guidelines for maximizing lifetime and avoiding energy holes in sensor networks with uniform distribution and uniform reporting. In: Proc. of IEEE INFOCOM , Apr. 2006.

11. Shi, Y., Thomas Hou, Y.: Approximation Algorithm for Base Station Placement in Wireless Sensor Networks. In Proc. IEEE Communications Society Conference on Sensor and Ad Hoc Communications and Networks (SECON), pp. 512-519, San Diego, CA, June 18-21, 2007.

12. Wang, W., Srinivasan, V., Chua, K.: Using mobile relays to prolong the lifetime of wireless sensor networks," in Proc. Of ACM MobiCom, Aug. 28 - Sept. 2, 2005.

13. Jea, D., Somasundara, A., Srivastava, M.: Multiple controlled mobile elements (data mules) for data collection in sensor networks. In IEEE DCOSS, pages 244--257, 2005.

14. Kinalis, A., Nikoletseas, S.: Scalable data collection protocols for wireless sensor networks with multiple mobile sinks. In 40th ANSS, pages 60-69, March 2007.

15. Luo, J., Hubaux, J. P.: Joint mobility and routing for lifetime elongation in wireless sensor networks In Proc. of IEEE INFOCOM, Mar. 2005.

16. Hashish, S., Karmouch, A.: Topology-based On-Board Data Dissemination Approach for Sensor Network. In ACM MobiWac 2007, Crete, Greece, October 22, 2007

[17] Sadler, B. M.: Fundamentals of energy-constrained sensor network systems. IEEE Aerospace and Electronic Systems Magazine, Tutorial Supplement, 2005.

18. Kansal, A., Rahimi, M., Kaiser, W. J., Srivastava, M. B., Pottie, G. J., Estrin, D.: Controlled mobility for sustainable wireless networks. In Proc., IEEE Sensor and Ad Hoc Communications and Networks (SECON), Santa Clara, CA, Oct. 2004.

19. Fang Q., Gao, J., Guibas L.: Locating and Bypassing Routing Holes in Sensor Networks. In 23rd Conference of the IEEE Communications Society (InfoCom), 2004.

20. Shamos M. I., Preparata, F.P.: Computational Geometry. Springer-Verlag, Berlin, 1985.

21. http://www.djstein.com/projects/WirelessSensorNetworkSimulator.html 